\title{
Swirling Flow Field inside a Hollow Turbine Shaft for an Internal Cooling Air System
}

\author{
TADAHARU KISHIBE ${ }^{\mathrm{a}, *}$ and SHOJIRO KAJI ${ }^{\mathrm{b}}$ \\ ${ }^{a}$ Gas Turbine R\&D Center, Hitachi Ltd., Horiguchi 832-2, Hitachinaka-shi, Ibaraki-ken 312, Japan, \\ ${ }^{b}$ Department of Aeronautics and Astronautics, University of Tokyo, Tokyo, Japan
}

(Received 22 August 1997; In final form 27 August 1998)

\begin{abstract}
The swirling flow field in an internal cooling air system in which the fluid passes through an inducer, a hollow turbine shaft, and a cavity between two disks (referred to as a wheel space) is solved using computational fluid dynamics and the pressure fluctuations on the hollow shaft wall surface are measured.

The three-dimensional compressible Navier-Stokes equations are adopted and discretized by an implicit TVD scheme. The region of the cooling air system is divided into two computational domains: one from the inducer to the hollow shaft, and the other from the hollow shaft to the wheel space. In the analysis of the former computational domain, the roles of components such as inducer blades are shown. In the analysis of the latter, the existence of a rotating spiral vortex at the place where the swirling flow turns radially outward is shown and its characteristics are described.

The main part of the internal cooling air system of a gas turbine is used as an experimental apparatus. Pressure sensors are embedded axially and circumferentially in the hollow turbine shaft to measure unsteady wall pressures. The existence and characteristics of the rotating spiral vortex are confirmed experimentally. The pressure fluctuations due to instability in the rotating wall boundary layer, whose waves propagate both in the positive and negative directions of the shaft rotation, are captured.
\end{abstract}

Keywords: Rotating spiral vortex, Swirling flow, Hollow turbine shaft, Internal cooling air system, Numerical analysis, Experiment

\section{INTRODUCTION}

High thermal efficiency of gas turbines is dependent on high turbine inlet temperature, which is achieved by cooling the turbine blades and nozzle guide vanes. In some gas turbines, the air taken from the compressor is cooled outside of the casing and then introduced into hot components through a rotating hollow turbine shaft, where it is possible that the Coriolis force causes swirling flow. The swirling flow causes various phenomena unwelcome to the internal cooling air system such as a pressure drop and instability. Clarifying phenomena peculiar to the swirling flow is

* Corresponding author. Tel.: 81292765733 . Fax: 81292765647. 
therefore very important to improve the gas turbine performance.

Owen and Pincombe (1980) and Owen et al. (1985) have carried out experiments with flow visualization and laser-Doppler anemometry on a rotating cylindrical cavity with a radial inflow and outflow of fluid, and Chew et al. (1984) have presented numerical solutions for steady, axisymmetric, source-sink flow in the cavity. The focus of the papers was on the cavity between two disks, but not much attention was paid to the flow field in the hollow turbine shaft.

In this study, not only radial inflow in an inducer and radial outflow in a wheel space, but also the swirling flow field in the hollow turbine shaft were numerically solved. The primary emphasis of the experiments was on unsteady non-axisymmetric

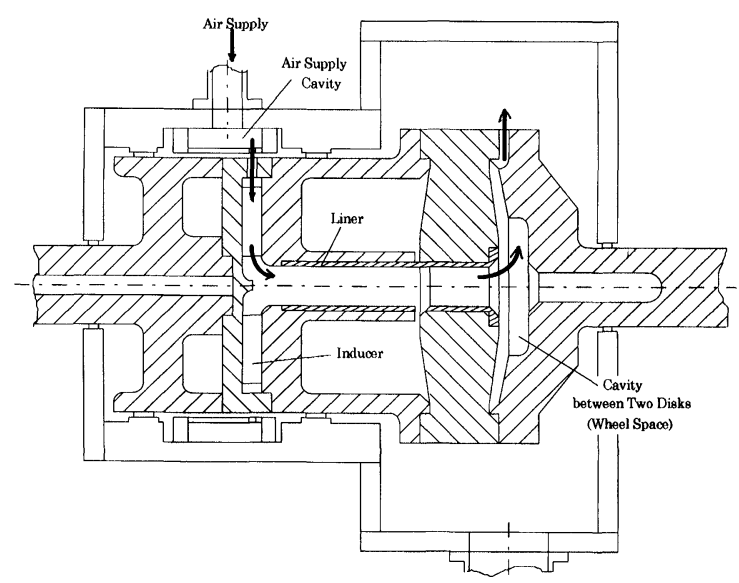

(a) In the r-z Plane

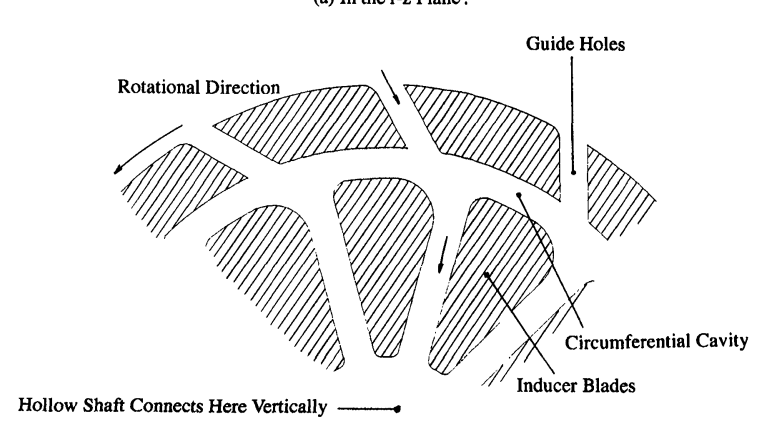

(b) For the Inducer in the r- $\theta$ Plane.

FIGURE 1 Cross section of the experimental apparatus. Arrows show flow directions. phenomena in the rotating shaft. A scale model of the internal cooling air system of an existing gas turbine was used as the experimental apparatus. Figure 1 shows schematic cross sections of the apparatus. The arrows show flow directions. The fluid passes through the inducer, the hollow shaft, where the liner for measurements is inserted, and the wheel space. Computational domains were based on the apparatus.

\section{NUMERICAL ANALYSES}

The three-dimensional compressible NavierStokes equations described in a relative cylindrical coordinate system rotating about $r=0$ were adopted and discretized by means of the implicit TVD scheme. Two subiterations per time step were made to reduce linearization and factorization errors for unsteady calculations. No eddy viscosity was used, since the vortex layer between the main swirling flow and the reversed flow is more important than the wall shear layer for the swirling flow in question and existing turbulence models are applicable to the flow field near the wall boundary layer. The molecular viscosity was calculated using Sutherland's law. Most of the boundary conditions were ordinary ones for subsonic viscous flow. In the analyses to find non-axisymmetric phenomena, axisymmetric boundary conditions along the center axis were avoided. These boundary conditions are explained in detail by Kishibe and Kaji (1995).

\section{From Inducer to Hollow Turbine Shaft}

The computational domain was from the inducer to the hollow shaft. The shape of the shaft was simplified to a straight one and the length was about five times the shaft diameter. Figure 2 shows the grid for the inducer in the $r-\theta$ plane. The inducer has 12 blades and 12 guide holes, so the computational domain was restricted to one periodic region of an angle of $30^{\circ}$ in the circumferential direction. Axisymmetric boundary conditions along the center axis of the hollow shaft, therefore, 


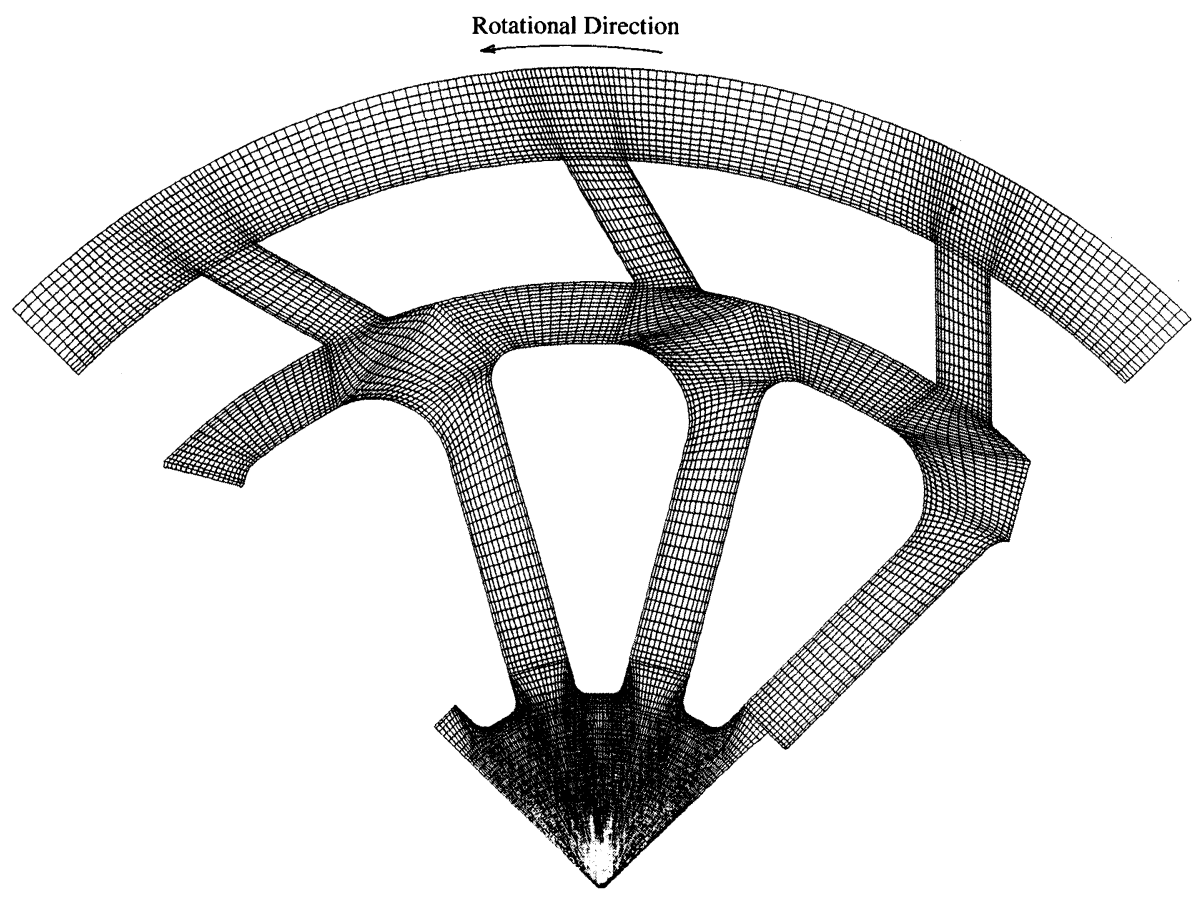

FIGURE 2 Grid for the inducer in the $r-\theta$ plane.

must be adopted. The cross section of the guide holes, which have a circular shape in the experimental apparatus, was simplified to a rectangular one in this analysis. This computational domain was so complicated that the zonal approach was adopted and the domain was divided into five regions. The grid lines on the zonal boundaries in the inducer overlapped each other to the extent of one grid point, except those between the inducer region and the hollow shaft region. The grid lines were not concentrated near walls any more than those used in general viscous numerical analyses. It should be noted that in this numerical analysis there was little possibility of capturing the unstable phenomena which may occur near the rotating wall.

Figure 3(a) plots velocity vectors in an $r-\theta$ plane in the rotating relative frame. The air through the guide holes does not directly enter paths between the inducer blades, but flows in the circumferential cavity in the direction opposite to the rotational direction of the shaft and then passes between the inducer blades. In the guide holes the flow field is not uniform and the reverse flow occupies a greater region. The flow field between the inducer blades, however, is uniform in comparison with that of the guide holes due to the circumferential cavity. The paths between the inducer blades are directed to the center axis in the hollow shaft. The flow in the hollow shaft, however, becomes swirling flow even in the rotating, relative frame due to the Coriolis force. The wakes of the inducer blades have a peculiarity that many wakes are concentrated. According to this analysis, however, the wakes do not generate any interesting phenomena. The flow field including the wakes quickly becomes uniform circumferentially as the wakes proceed downstream. Figure 3(b) plots the absolute total temperature contours. The paths between the inducer blades function efficiently; the cooling air is cooled (total temperature drop is $8^{\circ} \mathrm{C}$ for these computational conditions) and the energy taken out is returned as shaft power (as in a radial turbine).

It should be noted that further analyses with more grid points are needed in order to discuss details of the results obtained here and to capture 


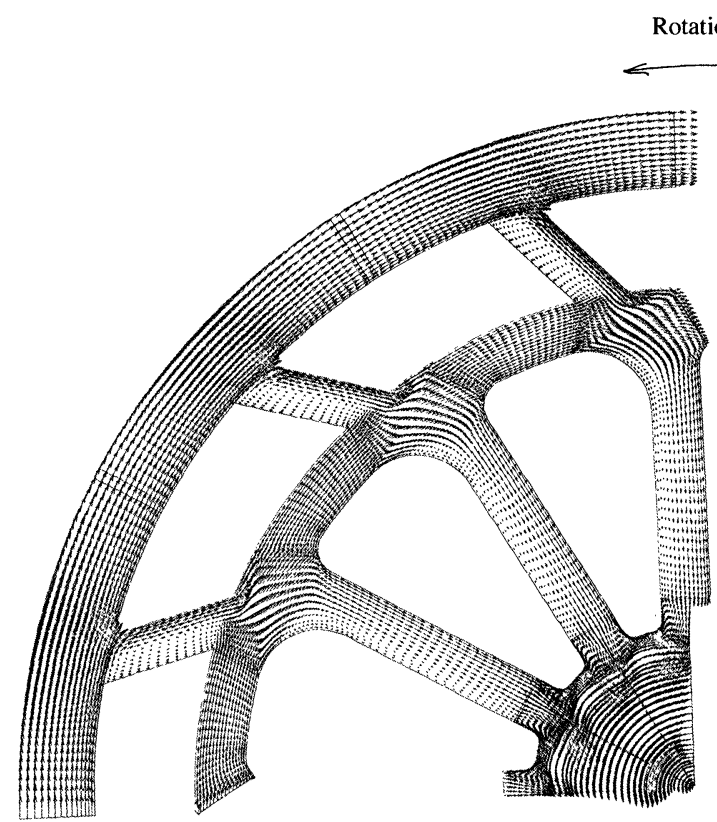

(a) Velocity Vectors . tational Direction

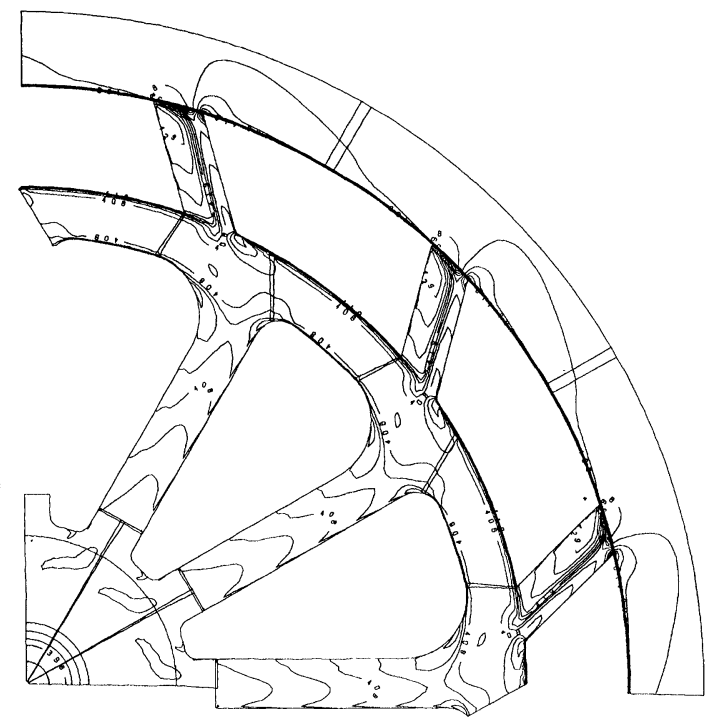

(b) Total Temperature Contours (unit: K) .

FIGURE 3 Flow field in the $r-\theta$ plane.

unstable phenomena which may occur near the rotating wall as stated above.

\section{From Hollow Turbine Shaft to Wheel Space}

In order to find non-axisymmetric phenomena, such as a spiral vortex, no axisymmetric assumption can be applied. The computational domain, therefore, was extended circumferentially to $360^{\circ}$ and axisymmetric boundary conditions along the center axis were avoided as done by Kishibe and Kaji (1995). The shape of the computational domain in the $r-z$ plane, then, must be simple and restricted. The domain was limited to the downstream region from the exit of the inducer, and the complicated geometry found in the internal cooling air system of an actual gas turbine was simplified. Assuming that non-axisymmetric phenomena appear in the vortex layer between the main swirling flow and the reversed flow, the grid lines were not concentrated near walls any more than those used in general viscous numerical analyses in order to decrease the number of grid points. It should be noted that the unstable phenomena which probably occur near the rotating wall may not be captured.

First, the computational domain was restricted to the region in the rotating hollow shaft (Fig. 4). This case has already been treated in detail by Kishibe and Kaji (1995), but the results are summarized below to help understand the numerical results for the swirling flow field extending from the rotating hollow turbine shaft to the wheel space.

A large-scale spiral vortex existed along the vortex layer between the main swirling flow and the reversed flow near the exit of the computational domain. The first non-axisymmetric mode of a single spiral vortex was transformed into the second mode of a double spiral vortex at a specific rotating speed of the shaft. The spiral vortex rotated about the shaft center axis in the same direction as the circumferential velocity of the main flow, which was the same direction as the rotating shaft. But, the vortex had a spiral form opposite to the rotational direction of the circumferential velocity of the main flow. 


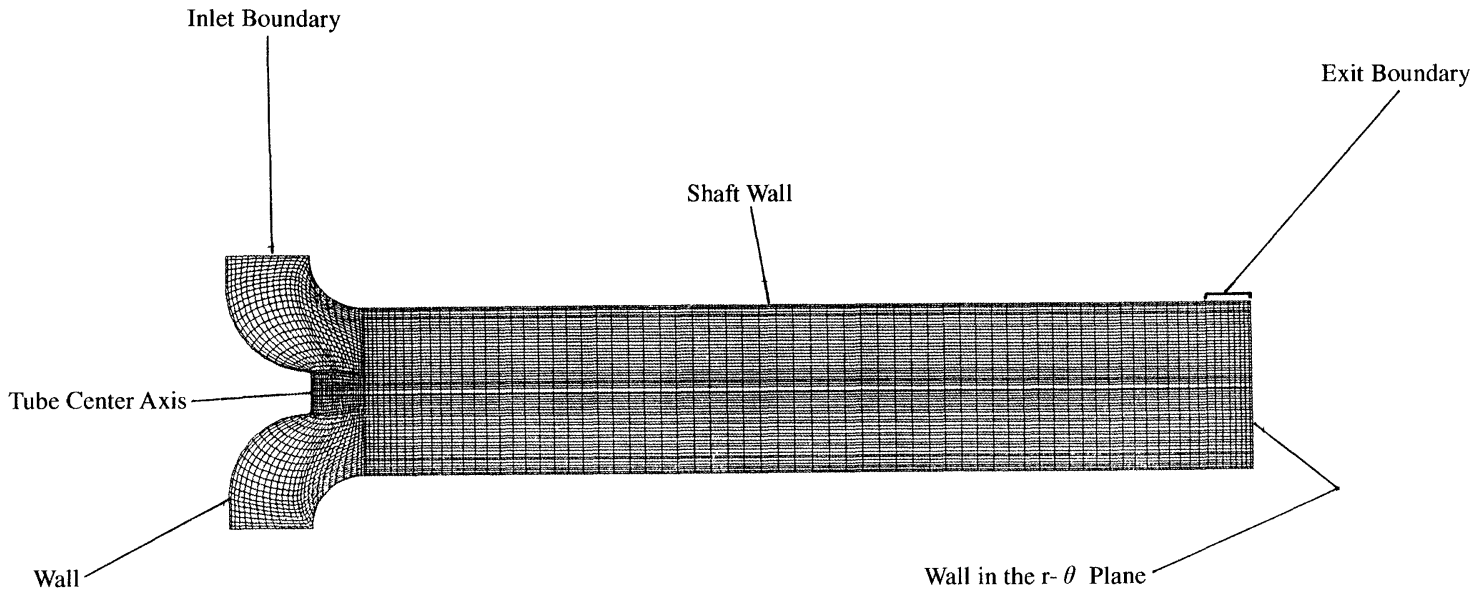

FIGURE 4 Grid in the $r-z$ plane.

The formation mechanism of the vortex could be described as follows. Though a zero relative circumferential velocity was imposed on the inlet boundary, the Coriolis force caused a swirling flow field. The pressure around the tube center axis was lower due to the centrifugal force. Since the angular momentum decreased downstream, the pressure around the center axis was lower upstream than downstream. A reverse flow therefore occurred along the center axis. Near the $r-\theta$ wall (turbine disk), where the swirling flow turned radially outward, a part of the flow turned toward the center axis due to the radial gradient of the pressure. This secondary flow grew into a large-scale vortex (Fig. 5). This vortex occurred uniformly along the entire circumference on the $r-\theta$ wall surface, thus the vortex was axisymmetric and doughnut-shaped. When the main flow included the circumferential component of velocity, the centerline of the doughnut vortex became loose and extended upstream, so that the doughnut shape became a spiral form. This was the rotating spiral vortex.

Chanaud (1965) and Nishi et al. (1982) showed experimentally that there was another rotating spiral vortex, which had the same characteristics as the above-mentioned spiral vortex, along the vortex layer in the upstream region of a swirling flow. This upstream vortex appeared clearly in the fluctuating flow field of these numerical results.

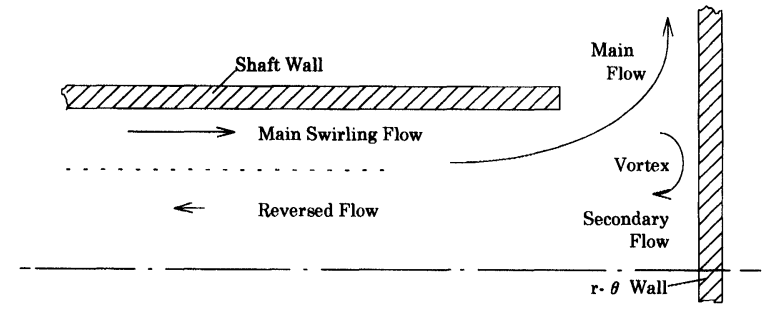

FIGURE 5 Schematic diagram of the vortex in swirling radial outflow.

Figure 6(a) shows the instantaneous distribution of the static pressure fluctuations in the $r-z$ plane. Along the upstream vortex layer there are waves whose amplitude is about one-fifth as large as that of the above-mentioned large-scale spiral vortex, which is seen near the exit. Figure 6(b) shows the axial distribution of the low pressure region in each cross section. A spiral vortex appears along the upstream vortex layer. This is the same type of rotating spiral vortex as seen by Chanaud and Nishi et al. Figure 6(b) includes the above-mentioned spiral vortex, which has twin spirals.

It was confirmed that the transition of the circumferential mode depends on the swirling rate $m$ defined as

$$
m=\int_{0}^{R} \frac{\rho v_{\theta} v_{z} r^{2}}{\mathrm{R}} \mathrm{d} r \int_{0}^{R} \rho v_{z}^{2} r \mathrm{~d} r
$$


(a)

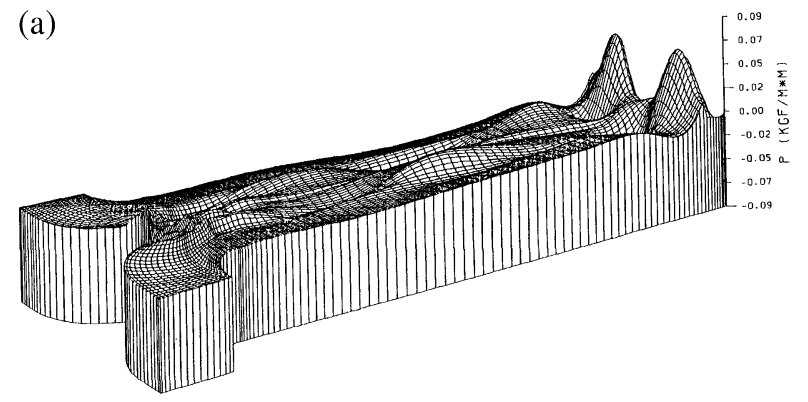

(b)
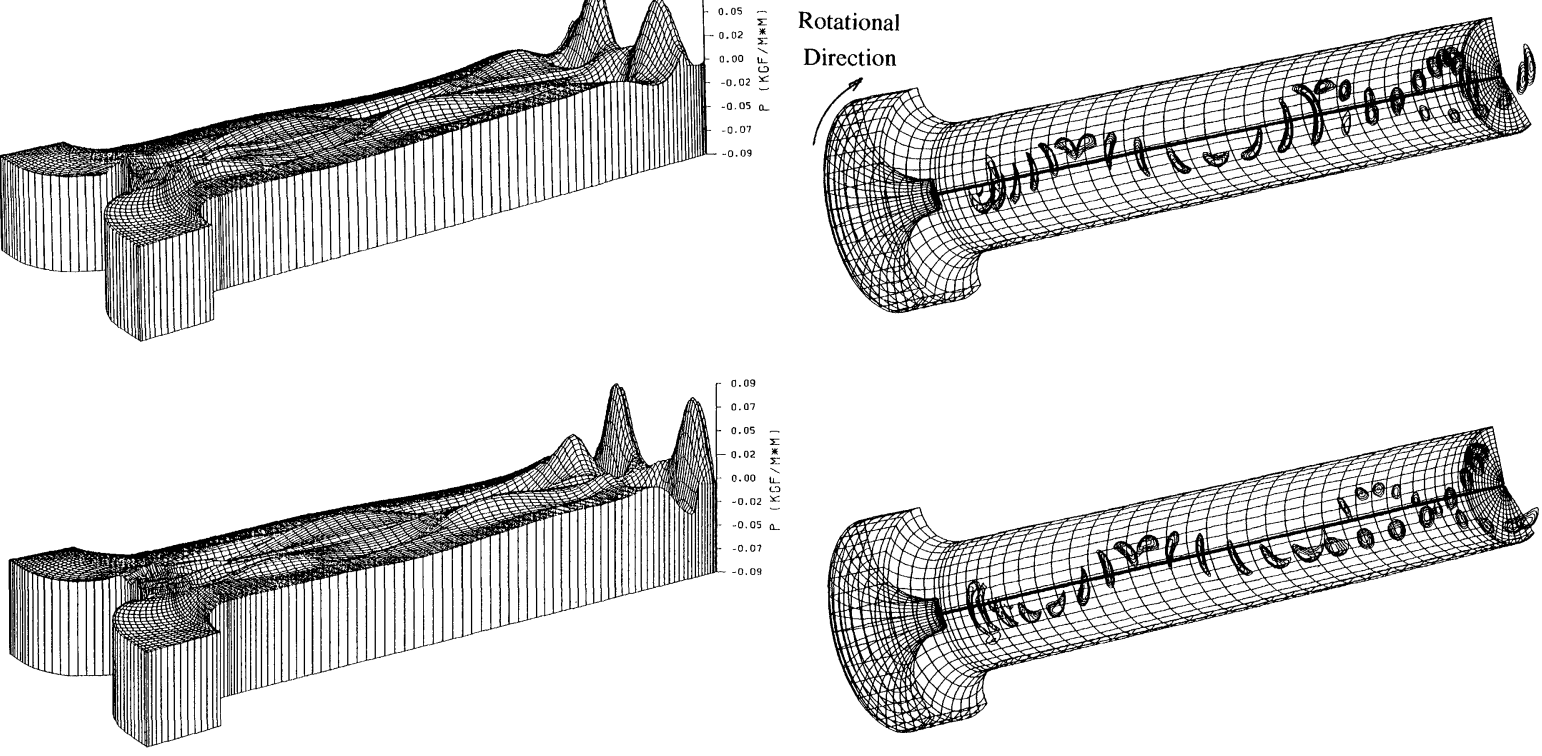

b)

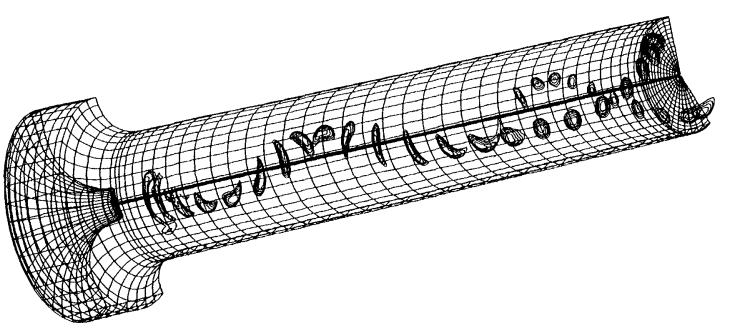

FIGURE 6 (a) Distribution of pressure fluctuations in the $r-z$ plane. The lower figure is $0.25 \mathrm{~ms}$ later than the upper figure. (b) Axial distribution of Low pressure regions. The lower figure is $0.25 \mathrm{~ms}$ later than the upper figure.

where $\rho, v_{\theta}, v_{z}$ and $R$ are the density, the circumferential velocity in the absolute frame, the axial velocity, and the inner radius of the shaft, respectively. The reason for the transformation of the spiral vortex is considered as follows: on increasing the swirling rate, the radius of the reversed flow region is increased, and the region of the main swirling flow is decreased accordingly as explained by Nishi et al. (1982). Since the static pressure on the exit boundary is fixed at a certain value in this numerical analysis, the radius of the spiral vortex, which exists in the vortex layer between the main swirling flow and the reversed flow, is restricted to a certain value even if the swirling rate is increased. The spiral vortex is consequently transformed into the double spiral on increasing the swirling rate. Then, the transition of the circumferential mode in this numerical analysis depends on the fixed static pressure on the exit boundary. The circumferential mode of the upstream spiral vortex, on the other hand, keeps the first mode at the swirling rate $m$ achieved in this analysis.
The downstream region of the computational domain was extended to the wheel space, the cavity between the corotating turbine disks (see Fig. 1), to avoid the above-mentioned influence of the fixed static pressure on the exit boundary. The data on precessing frequencies of the rotating spiral vortex in this numerical analysis were compared with experimental results. In addition, attention was paid to the three-dimensional swirling flow field in the rotating cavity with the rotating spiral vortex in the straight tube.

Figure 7 shows the grid in the $r-z$ plane, which is distributed uniformly in the circumferential direction. The swirling flow passes through the hollow shaft and leaves radially through a circumferential slit on the exit boundary. This computational domain is so complicated that the zonal approach was adopted. The grid lines on the zonal boundaries in two domains overlapped each other to the extent of one grid point. The parameters used in this analysis were set to allow comparison of the numerical results with experimental results. The inlet absolute total temperature was $288 \mathrm{~K}$. 


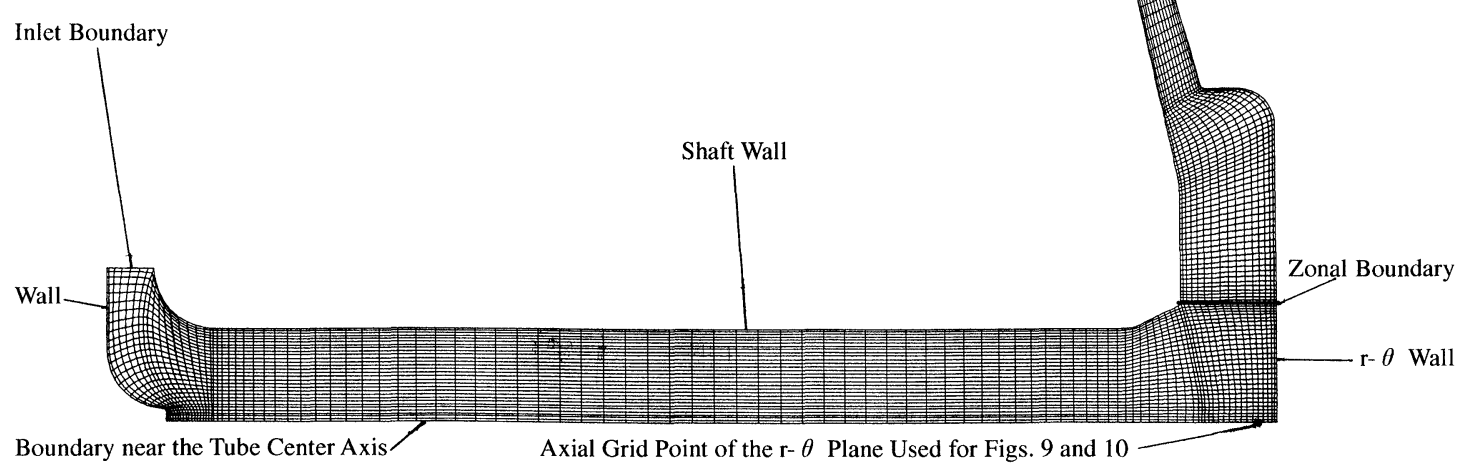

FIGURE 7 Grid in the $r-z$ plane.

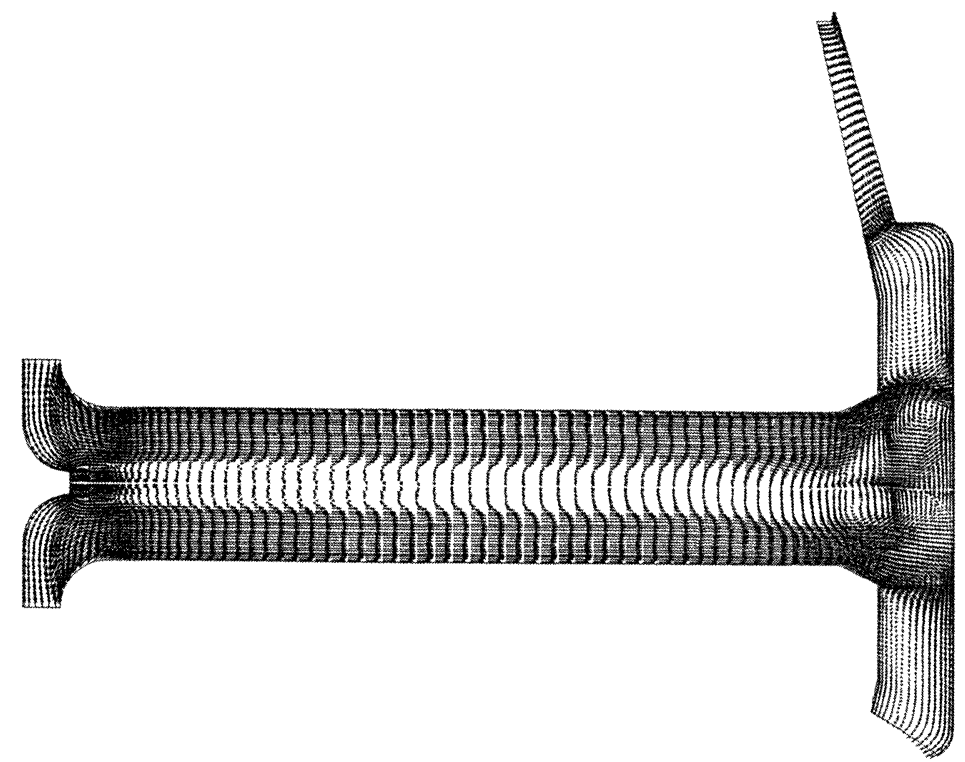

FIGURE 8 Velocity vectors in the $r-z$ plane.

The rotating speed was $5000 \mathrm{rpm}$. At this rotating speed, the exit static pressure was $0.265 \mathrm{MPa}$ and the mass flow rate was $1.03 \mathrm{~kg} / \mathrm{s}$.

Figure 8 plots the velocity vectors in the $r-z$ plane. Though the swirling rate was sufficiently large, the circumferential mode is not transformed into the second mode. Its character does not change even if the swirling rate was increased further by increasing the rotating speed or reducing the mass flow rate. 
The precessing frequency of the rotating spiral vortex in the numerical calculation is $420 \mathrm{~Hz}$. The frequency of pressure fluctuations is constant everywhere in the cavity between the disks. Figure 9 plots the static pressure contours at the axial grid point indicated in Fig. 7. Since the plane of the contours is curved in the $r-z$ plane as seen in Fig. 7, the contours are projected on the $r-\theta$ plane. Figure 9 includes two circles which show the zonal boundaries and the circle with the smallest radius in the circumferential grid lines. It also indicates two straight grid lines, whose angles are $0^{\circ}$ and $5^{\circ}$, to show the computational boundaries and specify the circumferential location of contour patterns easily. The previously mentioned spiral vortex appears as a low static pressure spot $\mathrm{L}_{1}$. There is another low static pressure spot $\mathrm{L}_{2}$ due to another spiral vortex, whose formation mechanism is the same as the spiral vortex reported by Chanaud (1965) and Nishi et al. (1982). It can be deduced as being generated in the shear layer on the tube wall in the swirling flow and going into the cavity between the disks. This pressure distribution rotates in a solid-rotation manner about $r=0$, and thus the frequency of the pressure fluctuations is constant everywhere in the cavity. Figure 10 shows the velocity vectors in the absolute frame in the same plane (the displayed region is wider radially than Fig. 9). The circumferential velocity distribution in the cavity between two disks is that of a forced vortex. The fluid in the cavity flows in the opposite direction to the rotational direction of the shaft. It is probable that the circumferential velocity of the fluid is peculiar to the swirling radial outflow in this cavity.

\section{EXPERIMENTS}

The main part of the internal cooling air system of a gas turbine was used as the experimental apparatus. The cross section of the apparatus was shown in Fig. 1. Techniques such as flow visualization or measurements with a pitot tube were difficult to apply in this apparatus so that a specially devised liner was inserted inside the hollow turbine shaft and ten pressure sensors were embedded axially and circumferentially in the liner to measure the

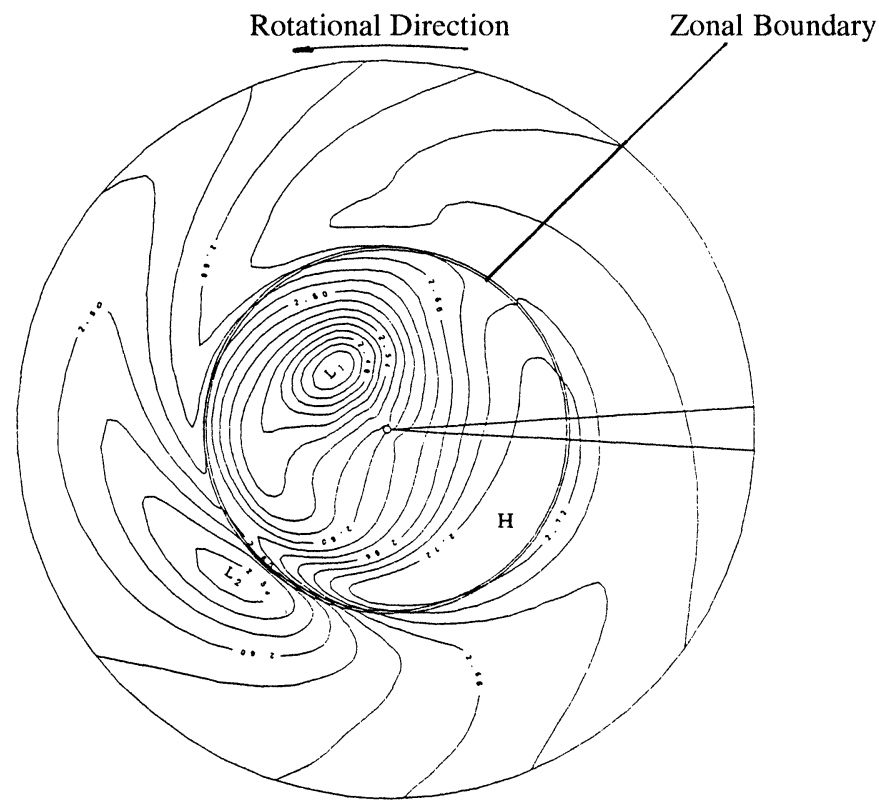

FIGURE 9 Static pressure contours in the cavity projected on the $r-\theta$ plane. $\mathrm{H}$ denotes high and $\mathrm{L}_{1-2}$ denotes low 


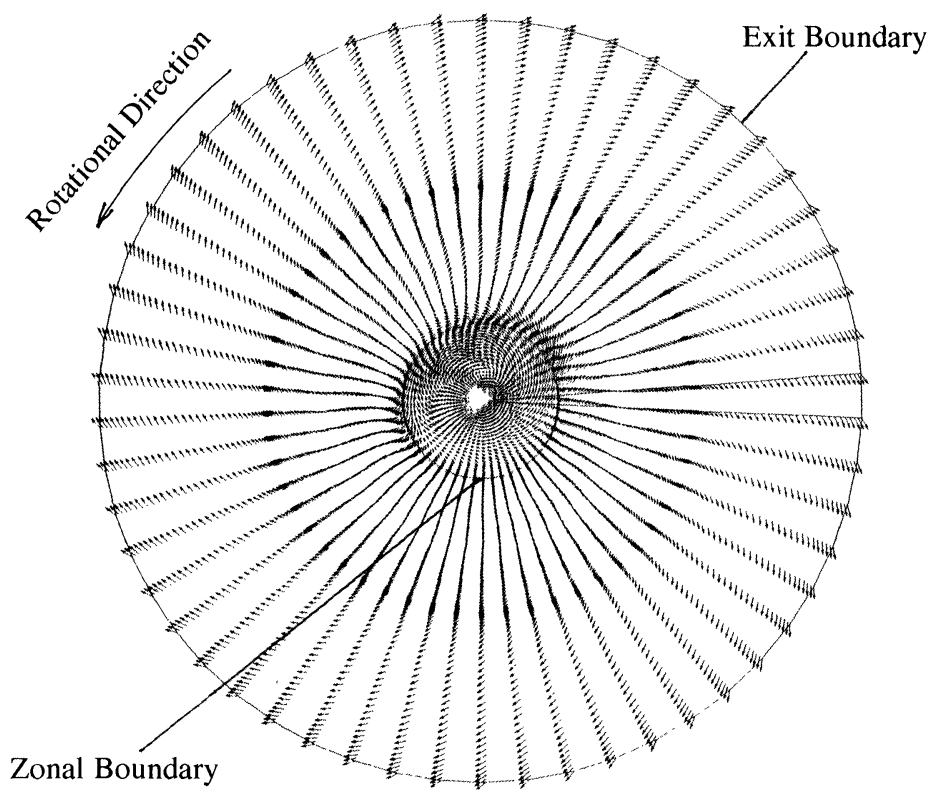

FIGURE 10 Velocity vectors in the same plane as Fig. 9. The display region is wider radially than Fig. 9.

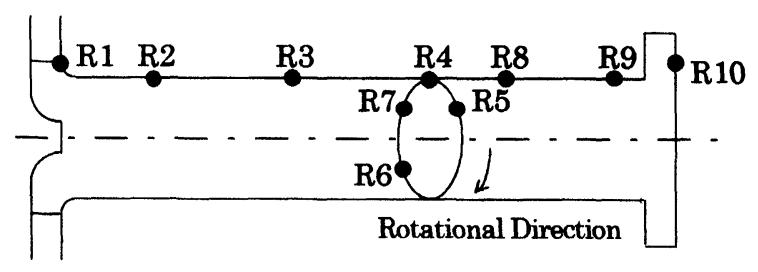

FIGURE 11 Location of pressure sensors R1-R10.

unsteady wall pressures. Figure 11 shows the locations of the pressure sensors R1-R10. Sensors $\mathrm{R} 4-\mathrm{R} 7$ were placed in the periphery of the $r-\theta$ cross section, but circumferentially apart. Sensors R1$\mathrm{R} 4$ and R8-R10 were arrayed axially at the same meridian. In addition to the sensors in the rotating frame, the pressure transducer $\mathrm{S} 1$ was set at the exit point of the air supply cavity. A slip-ring was used to send the outputs from the pressure sensors to amplifiers. The raw data from the amplifiers were recorded with a data-recorder and analyzed later. The precision of the measurement for phase difference between the sensors mainly depends on the accuracy of the synchronization between the channels of the data-recorder. Synchronization accuracy of the data-recorder used was less than
$15 \mu \mathrm{s}$. When the frequency was $400 \mathrm{~Hz}$, the phase difference error was $2.2^{\circ}$. The pressure sensors rotated with the shaft, and in the above-mentioned numerical analysis the pressure data were obtained at points fixed in the rotating coordinates system. No conversion of frequency was necessary to compare the frequency measured in the experiments with that of the numerical results.

The experiments were performed over a wide range of parameters. The parameters described here, however, are restricted to the same values as in the numerical analyses. Figure 12 shows the frequency spectra at shaft rotating speed $=4987 \mathrm{rpm}$, mass flow rate $=1.0 \mathrm{~kg} / \mathrm{s}$, and the total pressure in the upstream cavity to supply the fluid $=$ $0.294 \mathrm{MPa}$.

\section{Dominant Pressure Fluctuations in Downstream Region}

Attention is given to the peaks at $460 \mathrm{~Hz}$. The pressure fluctuations have the following characteristics.

(i) The peaks of the pressure fluctuations appear at sensors downstream from the sensor R4, 

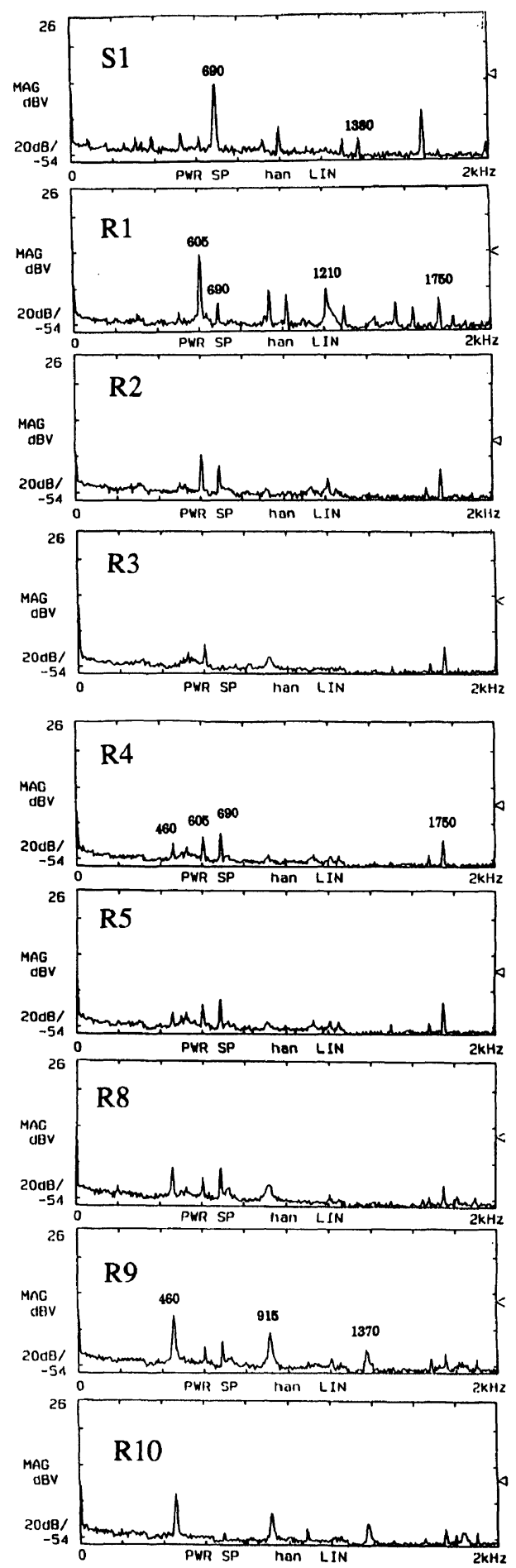

FIGURE 12 Frequency Spectra Showing $\mathrm{Hz}$ values of Selected Peaks. S1 is the transducer on a stationary component and R1-R10 are sensors given in Fig. 11.
TABLE I (a) Circumferential phase difference

\begin{tabular}{lrc}
\hline Sensor & Angle & Phase difference \\
\hline R4 & $0^{\circ}$ & $0^{\circ}$ \\
R5 & $60^{\circ}$ & $-55^{\circ}$ \\
R6 & $240^{\circ}$ & $-248^{\circ}$ \\
R7 & $300^{\circ}$ & $-309^{\circ}$ \\
\hline
\end{tabular}

TABLE I (b) Axial phase difference

\begin{tabular}{lcc}
\hline Sensor & Axial location $(\mathrm{mm})$ & Phase difference \\
\hline R4 & 0 & $0^{\circ}$ \\
R8 & 55 & $-47^{\circ}$ \\
R9 & 135 & $-104^{\circ}$ \\
R10 & 175 & $-304^{\circ}$ \\
\hline
\end{tabular}

and the peaks become larger as the fluid flows downstream. At higher swirling rate, realized by increasing the rotating speed or reducing the mass flow rate, the pressure fluctuations at the sensor R9 become larger.

(ii) Table I shows the circumferential and axial phase differences of sensors relative to the sensor R4. Negative values mean that the phase of the sensor lags behind that of R4. The circumferential phase difference is approximately equivalent to the angle between the sensors, the circumferential phase progresses opposite to the rotational direction of the shaft, and the axial phase is delayed more as the fluid flows downstream. The waves of the pressure fluctuations are thought to form the single spiral vortex, which rotates in the same direction as the rotating shaft, and to lead to the spiral form which moves opposite to the rotational direction of the shaft.

The pressure fluctuations generated by a rotating spiral vortex were measured. The measured amplitude is great at sensors near the place where the vortex existed in the numerical results and the precession frequency of the rotating spiral vortex is in close agreement with the calculated frequency. It can be concluded that the pressure fluctuations measured are generated by the rotating spiral vortex, which is predicted numerically. 


\section{Dominant Pressure Fluctuations in Upstream Region}

The peaks at $605 \mathrm{~Hz}$ in Fig. 12 are discussed in this section. The pressure fluctuations have the following characteristics.

(i) The peaks of the pressure fluctuations appear at almost all sensors, except sensor R10. The most upstream sensor R1 has the largest peak, and the peaks become smaller as the fluid flows downstream.

(ii) The circumferential or axial phase difference between the sensors is not detected in the experimental results shown in Fig. 12. At some experimental parameters, however, the phase difference is found. The circumferential phase difference is nearly equal to the installation angle between the sensors, and the circumferential phase progresses coincident with the rotational direction of the shaft. The axial phase progresses as the fluid flows downstream. These characteristics are opposite to those of the peaks at $460 \mathrm{~Hz}$.

(iii) For some sensors the peaks at $690 \mathrm{~Hz}$ and/or $520 \mathrm{~Hz}$ can be seen. The difference between these frequencies and $605 \mathrm{~Hz}$ is the rotating speed of the shaft $(83 \mathrm{~Hz}$ at $5000 \mathrm{rpm})$. At the transducer S1 in Fig. 12 the peak at the positively shifted frequency is greater than that of the negatively shifted frequency, but at some experimental parameters the opposite tendency is observed. It seems that this behavior is not fixed.

It can be deduced that characteristic (iii) is due to waves of an unstable phenomenon occurring in a rotating wall boundary layer which propagate in the same, as well as the opposite, direction of the shaft rotation. This unstable phenomenon, however, could not be specified. Although the amplitude of the pressure fluctuations is no less than about $10 \%$ of the supplied air pressure, the pressure fluctuations could not be captured in the numerical analyses. Thus, further numerical analyses and experiments are needed to clarify the pressure fluctuations.

\section{CONCLUSIONS}

Numerical results were presented for swirling flow fields in an internal cooling air system.

In the inducer region, the results described below were obtained. In the guide holes the flow field was not uniform and the reverse flow occupied a greater region, but the flow field between the inducer blades was uniform due to the circumferential cavity. The cooling air was cooled and the energy taken out was returned as shaft power through the paths between the inducer blades. When the paths between the inducer blades were directed to the center axis in the hollow shaft, the flow in the hollow shaft became swirling flow due to the Coriolis force. The flow field, including the wakes of the inducer blades, quickly became uniform circumferentially as the wakes flowed downstream.

In a hollow turbine shaft, a large-scale spiral vortex existed along the vortex layer between the main swirling flow and the reversed flow at the place where the swirling flow turned radially outward. The spiral vortex rotated about the shaft center axis in the same direction as the circumferential velocity of the main flow. Conversely, the spiral vortex had a spiral form opposite to the rotational direction of the fluid.

In the wheel space, the cavity between the corotating disks, there was another spiral vortex, which could be deduced as generated in the tube wall shear layer in the swirling flow and flowing into the cavity. The frequency of pressure fluctuations in the cavity was constant everywhere. The circumferential velocity in the cavity was that of a forced vortex.

In the experiments, unsteady wall pressures inside the turbine shaft were measured.

The pressure fluctuations which had the same characteristics as the rotating spiral vortex predicted in the numerical results were measured. The amplitude was great at the sensors near the place where the vortex was predicted in the numerical results and the precession frequency of the rotating spiral vortex was in close agreement with the calculated frequency. The existence and 
characteristics of the rotating spiral vortex which appeared in the numerical results were thus confirmed experimentally.

At some sensors some peaks appeared at positively and/or negatively shifted frequencies by the rotating speed of shaft. It could be deduced that the pressure fluctuations were due to an unstable phenomenon in the rotating wall boundary layer, whose waves propagated in the rotational direction of the shaft and also in the opposite direction.

\section{Acknowledgments}

The authors wish to thank M. Matsumoto, T. Ikeguchi and I. Takehara of Hitachi Ltd. for their help in conducting the experiments. The authors would like to thank M. Hiramoto of University of Tokyo for his overall help in this research work. The authors are especially grateful for support of the computational work given by the National Aerospace Laboratory.

\section{References}

Chanaud, R.C., 1965. Observations of oscillatory motion in certain swirling flows, J. Fluid Mech., 21, 111-127.

Chew, J.W Owen, J M and Pincombe, J R 1984. Numerical predictions for laminar source-sink flow in a rotating cylindrical cavity, J. Fluid Mech., 143, 451-466.

Kishibe, T. and Kaji, S., 1995. Study on the flow field of an internal cooling air system through a hollow turbine shaft, I.G.T.C. Yokohama, Vol. II, pp. 383-391.

Nishi, M., Kubota, T., Matsunaga, S. and Senoo, Y., 1982. Study on pressure surge and swirl flow in the elbow type draft tube of a francis turbine, J.J.S. M. E., 48(431), 1238-1246 (in Japanese).

Owen, J.M. and Pincombe, J.R., 1980. Velocity measurements inside a rotating cylindrical cavity with a radial outflow of fluid, J. Fluid Mech., 99, 111-127.

Owen, J.M., Pincombe, J.R. and Rogers, R.H., 1985. Sourcesink flow inside a rotating cylindrical cavity, J. Fluid Mech., $155,233-265$ 


\section{ait \\ ENERGY MATERIALS}

M A N E Y publishing

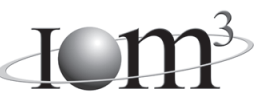

\section{Materials Science \& Engineering for Energy Systems}

Maney Publishing on behalf of the Institute of Materials, Minerals and Mining

The Institute of Materials, Minerals \& Mining

Economic and environmental factors are creating ever greater pressures for the efficient generation, transmission and use of energy. Materials developments are crucial to progress in all these areas: to innovation in design; to extending lifetime and maintenance intervals; and to successful operation in more demanding environments. Drawing together the broad community with interests in these areas, Energy Materials addresses materials needs in future energy generation, transmission, utilisation, conservation and storage. The journal covers thermal generation and gas turbines; renewable power (wind, wave, tidal, hydro, solar and geothermal); fuel cells (low and high temperature); materials issues relevant to biomass and biotechnology; nuclear power generation (fission and fusion); hydrogen generation and storage in the context of the 'hydrogen economy'; and the transmission and storage of the energy produced.

As well as publishing high-quality peer-reviewed research, Energy Materials promotes discussion of issues common to all sectors, through commissioned reviews and commentaries. The journal includes coverage of energy economics and policy, and broader social issues, since the political and legislative context influence research and investment decisions.

\section{CALL FOR PAPERS}

Contributions to the journal should be submitted online at http://ema.edmgr.com

To view the Notes for Contributors please visit: www.maney.co.uk/journals/notes/ema

Upon publication in 2006, this journal will be available via the Ingenta Connect journals service. To view free sample content online visit: www.ingentaconnect.com/content/maney

For further information please contact:

Maney Publishing UK

Tel: +44 (0)113 2497481 Fax: +44 (0)1132486983 Email: subscriptions@maney.co.uk

or

Maney Publishing North America

Tel (toll free): 8662975154 Fax: 6173546875 Email: maney@maneyusa.com

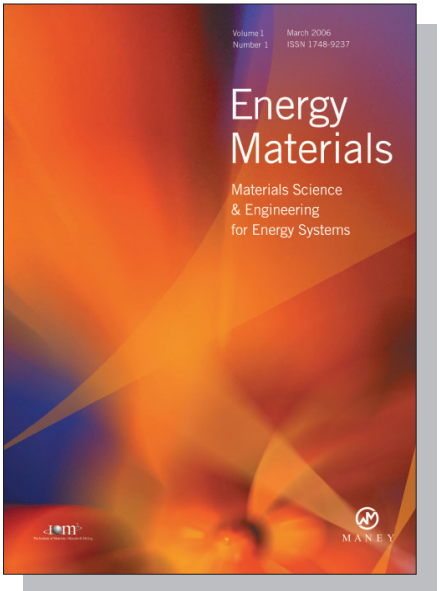

EDITORS

Dr Fujio Abe

NIMS, Japan

Dr John Hald, IPL-MPT, Technical University of Denmark, Denmark

Dr R Viswanathan, EPRI, USA

\section{SUBSCRIPTION INFORMATION}

Volume 1 (2006), 4 issues per year

Print ISSN: 1748-9237 Online ISSN: 1748-9245

Individual rate: $£ 76.00 / U S \$ 141.00$

Institutional rate: $£ 235.00 /$ US $\$ 435.00$

Online-only institutional rate: $£ 199.00 / U S \$ 367.00$

For special $\mathrm{IOM}^{3}$ member rates please email

subscriptions@maney.co.uk

\section{For further information or to subscribe online please visit www.maney.co.uk}



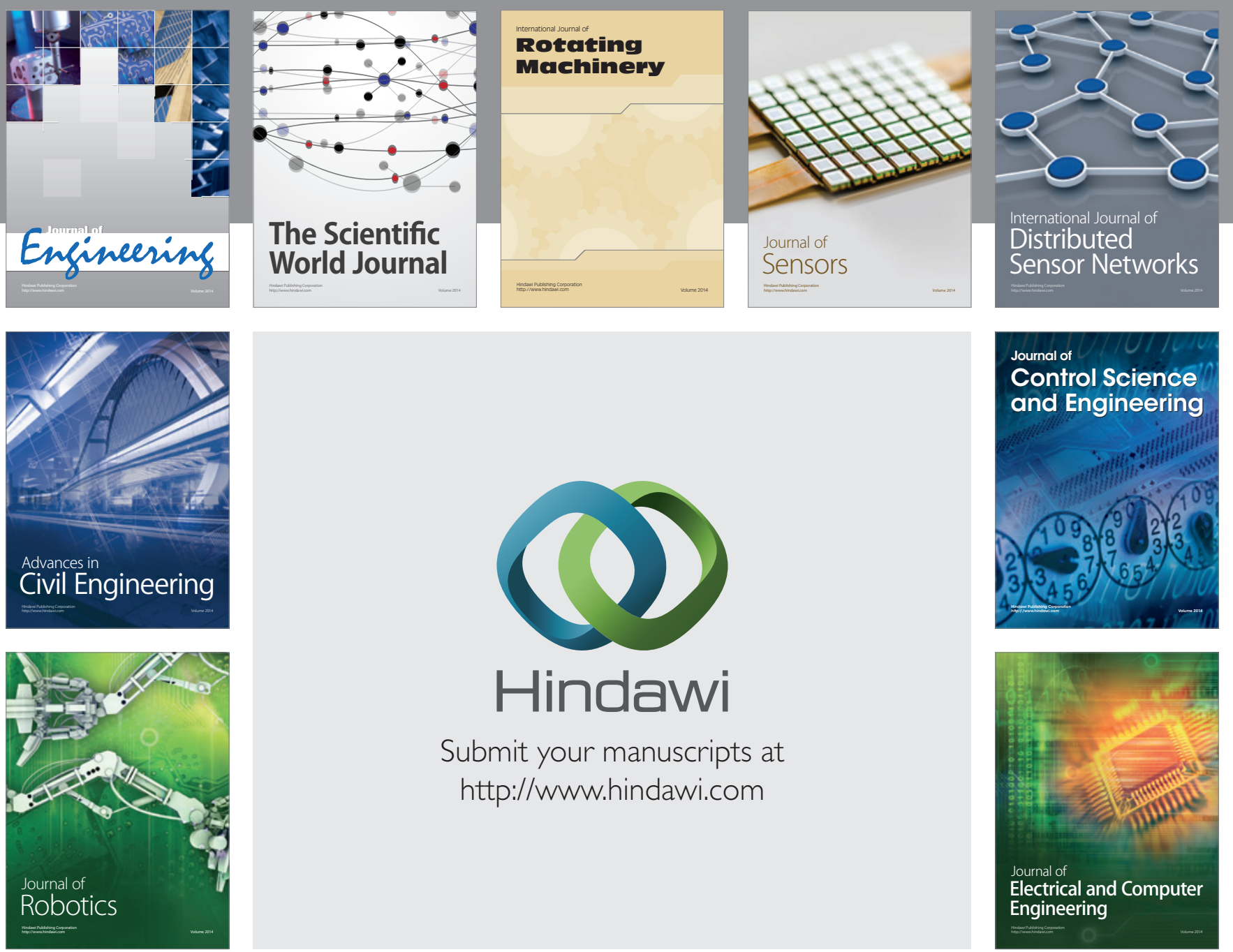

Submit your manuscripts at

http://www.hindawi.com
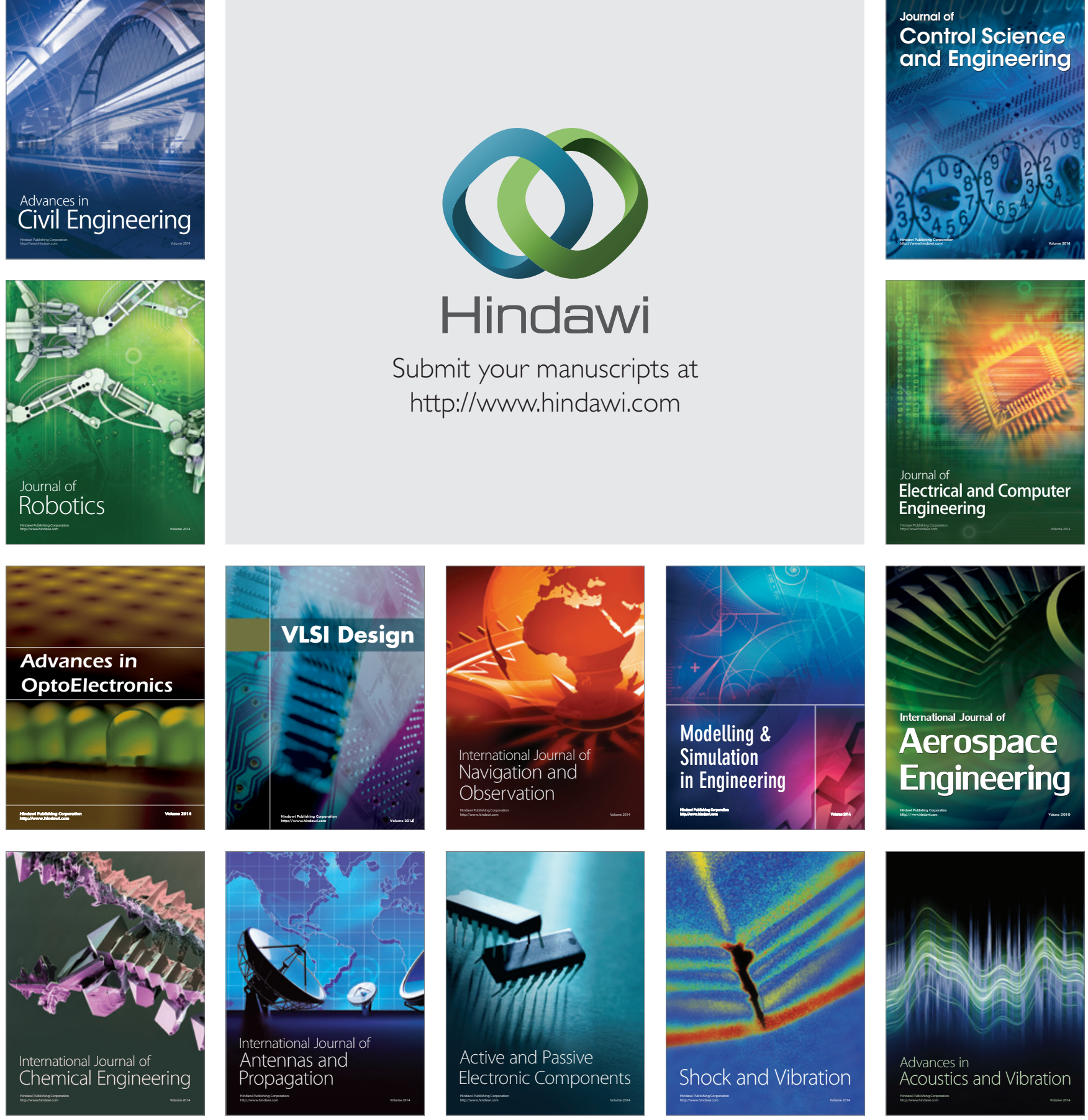